\title{
Activation of Selected Core Muscles during Pressing
}

\author{
Thomas W. Nesser (Corresponding author) \\ Kinesiology, Recreation, and Sport, Indiana State University 401 N. 4th Street, Terre Haute, IN 47809 USA \\ E-mail: tom.nesser@indstate.edu \\ Neil Fleming \\ Kinesiology, Recreation, and Sport, Indiana State University 401 N. 4th Street, Terre Haute, IN 47809 USA \\ E-mail: neil.fleming@indstate.edu \\ Matthew J. Gage \\ Department of Health Professions, Liberty University 1971 University Blvd, Lynchburg, VA 24515 USA \\ E-mail: mjgage@liberty.edu
}

Received: 28-09- 2015

Accepted: 20-10- 2015

Published: 31-10- 2015

doi:10.7575/aiac.ijkss.v.3n.4p.56

URL: http://dx.doi.org/10.7575/aiac.ijkss.v.3n.4p.56

\begin{abstract}
Introduction: Unstable surface training is often used to activate core musculature during resistance training. Unfortunately, unstable surface training is risky and leads to detraining. Purpose: The purpose of this study was to determine core muscle activation during stable surface ground-based lifts. Methods: Fourteen recreational trained and former NCAA DI athletes (weight $84.2 \pm 13.3 \mathrm{~kg}$; height $176.0 \pm 9.5 \mathrm{~cm}$; age $20.9 \pm 2.0$ years) volunteered for participation. Subjects completed two ground-based lifts: overhead press and push-press. Surface EMG was recorded from 4 muscles on the right side of the body (Rectus Abdominus (RA), External Oblique (EO), Transverse Abdominus (TA), and Erector Spinae (ES). Results: Paired sample T-tests identified significant muscle activation differences between the overhead press and the push-press included ES and EO. Average and peak EMG for ES was significantly greater in push-press $(\mathrm{P}<0.01)$. Anterior displacement of COP was significantly greater in push-press compared to overhead press during the eccentric phase. Conclusion: The push-press was identified as superior in core muscle activation when compared to the overhead pressing exercise.
\end{abstract}

Keywords: torso, stability, weight lifting, resistance training

\section{Introduction}

\subsection{Introduce the Problem}

The use of core training in the conditioning of both athletes and non-athletes has increased in the last decade (Gamble, 2007). Core training is defined as any exercise which utilizes motor control and muscular capacity of the lumbo-pelvic complex (Leetun, 2004; Gamble, 2007). The muscles of the core typically include the rectus abdominus, external and internal obliques, transverse abdominus, and erector spinae (McGill et al. 2003). The popularity of core training is based on the belief that a strong core allows greater spine stability and more effective transfer of forces from the lower body to the upper body with minimal dissipation of energy (Bompa, 1999; McGill, 2009) leading to an improvement in athletic performance such as higher jumps and faster sprints (Akuthota, 2004; Kibler,2006; King, 2000; Mayhew, 2005; McGill 1999), and reduced risk of lower limb injury (Leetun et al., 2004).

\subsection{Explore Importance of the Problem}

A current challenge is how best to train the muscles of the core. Basic core exercises include floor planks which require the maintenance of a prone position balanced on the elbows and toes. Floor planks activate core muscle but from a sport performance perspective, they are static and have little transfer to the sports arena (Parkhouse \& Ball, 2011). To step up the training intensity, planks have been completed on an unstable surface with increased EMG core muscle activation (Byrne et al. 2014; Snarr \& Esco, 2014), though the lack of sport specificity still applies. To train the core in a more sport specific manner, free weight exercises have been completed on an unstable surface. For example, completing a bench press while lying on a Swiss ball or standing on a foam pad during an overhead press. Training on an unstable surface does require more balance, unfortunately, unstable surface training is not ideal.

\subsection{Relevant Scholarship}

Several Electromyography (EMG) studies have been completed to confirm unstable training can increased core muscle activation (Anderson \& Behm, 2005, Norwood et al, 2007, Marshall \& Murphy, 2006) though not all core muscle EMG studies agree. Some identified greater core muscle activation on a stable vs. an unstable surface (Willardson et al., 2009, Kohler et al.,2010, Hamlyn et al., 2007), while others identified no difference between stable and unstable conditions (Gullett et al., 2009), Uribe et al., 2010, Saeterbalken \& Fimland, 2013). Likewise, Saeterbalken \& Fimland, (2013), 
Willardson, (2007), Behm et al. (2010), and Hamlyn et al.( 2007), identified reduced force output when training on an unstable surface which can lead to detraining.

Along with the possibility of detraining, unstable surface training is not practical. As identified by Kohler et al. (2010), most athletes compete on a "stable surface" (i.e. the ground). Thus the use of an unstable environment is not sport specific reducing the transfer of training to the field. Ground based lifts are most specific to sport due to the stabilization of an external load (barbell or dumbbell) on a stable surface (the ground) much like an athlete stabilizing an implement or opponent.

For practical and sport specific purposes, Behm et al. (2010) suggest the use of "ground-based lifts" such as "Olympic lifts, squats, and dead lifts" as a means to train the muscles of the core. Ground-based lifts are defined as lifts completed while in a standing position requiring the transfer of forces from the ground to the body.

Hamlyn et al. (2007) did just that by examining muscle activity of the upper lumbar erector spinae (UES), lumbar-sacral erector spinae (LES), lower abdominals (LA), and external obliques (EO) during two ground-based lifts, the back squat and the deadlift, with $80 \%$ 1-RM, plus three non-ground-based lifts: a bodyweight squat (no external load), a superman and a sidebridge. The two ground-based lifts generated greater UES and LES activity and similar LA and EO activity when compared to the other exercises suggesting ground-based lifts are more effective at core muscle activation than non-ground-based exercises.

\subsection{Hypotheses and Research Design}

Based on the results of the research identified, ground-based lifts are not only ideal but recommended for core muscle training. However, core muscle activation has not been compared between various ground-based lifts. Therefore, the first purpose of this study was to determine the magnitude of core muscle activation generated during commonly performed ground-based lifts such as the overhead press and push-press, and the second purpose was to determine if one exercise variation generated greater core muscle activation than the other. The push-press was hypothesized to generate greater muscle activation of all muscles measured with a forward shift in the center of pressure when compared to the overhead press due to the increased dynamics of the push-press.

\section{Methods}

\subsection{Experimental Approach}

This study was designed to compare core specific muscle activation between various multi-joint resistance training exercises. The dependent variables included activation of the right side rectus abdominus, external oblique, transverse abdominus, and erector spinae.

\subsection{Participants}

Fourteen recreational trained and former NCAA DI athletes (weight $84.2 \pm 13.3 \mathrm{~kg}$; height $176.0 \pm 9.5 \mathrm{~cm}$; age $20.9 \pm$ 2.0 years) volunteered for participation. Sample size was based on previous EMG studies (Anderson et al., 2004; Barnett et al., 1995; Gullett et al., 2009; Marshall \& Murphy, 2006; Norwood et al., 2007). All participants were free of injury at the time of data collection. All participants signed informed consent forms prior to participation. The university institutional review board approved this study.

\subsection{Procedures}

All subjects were individually scheduled for data collection. The press exercises were completed random order. All subjects were educated on the procedures and expectations of the research study. Prior to data collection all subjects practiced each exercise with minimal resistance to avoid fatigue. Each data collection session was completed within one hour.

\subsection{Overhead Press}

Subjects removed a weighted barbell from a squat rack and position it across the anterior deltoids with a closed pronated grip. Upon command, the subjects pressed the weight overhead with no movement of the lower body. Once the elbows were fully extended the bar was returned to the shoulders and repeated two more times. Subjects completed one set of three repetitions with 50 percent body mass.

\subsection{Push-press}

Subjects removed a weighted barbell from a squat rack and position it across the anterior deltoids with a pronated grip similar to the overhead press. Upon command, the subjects dipped down by slightly flexing the knees and then forcefully extended the knees just prior to pressing the weight overhead to generate momentum. Once the elbows were fully extended the bar was returned to the shoulders and repeated two more times. Subjects completed one set of three repetitions with 50 percent body mass.

\subsection{Surface Electromyography}

Surface EMG data were recorded from 4 core muscles on the right side of the body; Erector Spinae (ES), Rectus Abdominus (RA), External Oblique (EO), and Transverse Abdominus (TA). Data were collected using a Trigno wireless EMG data acquisition system (Delsys, Boston, MA, USA). Surface electrodes had a single differential configuration, inter-electrode distance of $10 \mathrm{~mm}, 4$-bar formation, bandwidth of $20-450 \mathrm{~Hz}$ and $99.9 \%$ silver contact material. All efforts were made to conform to the recommendations of SENIAM with regard to preparation and acquisition of EMG signals (Hermens et al., 2000). The skin sites were shaved and cleaned with isopropyl alcohol in order to minimize skin impedance. Data sampling rate was $2,000 \mathrm{~Hz}$ throughout all trials. The electrodes for RA were placed $1 \mathrm{~cm}$ above the umbilicus and $2 \mathrm{~cm}$ lateral to the midline. For EO, electrodes were placed below the ribcage, 
along a line between the most inferior point of the costal margin and the contra-lateral pubic tubercle. ES electrodes were placed $5 \mathrm{~cm}$ lateral to the level of the T9 spinous process. TA electrodes were placed $2 \mathrm{~cm}$ medial and inferior to the anterior superior iliac spine (ASIS). This site is below the EO fibers, thus reducing risk of cross-talk for this muscle.

Maximal voluntary isometric contractions (MVIC) were performed in order to normalize EMG data to a maximal reference for each muscle. MVIC procedures for RA, EO and TA, involved subjects lying supine on a table with legs fixed in place. A resisted sit-up from this position was performed for RA. Subjects were instructed to raise their torso off the table with maximal force while investigators held both shoulders in place. A resisted transverse sit-up was performed for EO normalization. Subjects were instructed to raise their right shoulder off the table towards their left hip, while investigators held the right shoulder in place. To measure MVIC for the TA, subjects were instructed to maximally pull their stomach in toward the spine. For ES normalization, subjects lay in a prone position and were instructed to raise their torso off the table with maximal force while the investigators held both shoulders in place.

Synchronous measurements of right knee, hip and elbow joint angle were made using electrogoniometers (Biometrics Ltd. $2000 \mathrm{~Hz}$ recording frequency) during all exercises. Data were collected in order to identify the onset of each movement cycle and differentiate the concentric and eccentric phases of the movement. Onset of overhead press and push-press were defined as first extension of the elbow or first flexion of the knee (in the case of push-press).

Following synchronization of EMG with the movement cycle, the root mean square (RMS) values of the raw data were calculated (50 ms window, 0 overlap). For each movement cycle, mean activity for the concentric phase, eccentric phase and overall movement cycle were calculated. These data were then averaged over 3 cycles and expressed as a percentage of MVIC.

\subsection{Center of Pressure Data}

Center of pressure data were measured during all lifts using a Tekscan HR mat (Tekscan, Boston, MA) in order to assess anterior displacement of the subject's center of pressure (COP) during each movement. The Tekscan HR mat has a surface area of $2323 \mathrm{~cm}^{2}$ (dimensions: $48.7 \times 47.7 \mathrm{~cm}$ ), a sensor resolution of 4 sensels $/ \mathrm{cm}^{2}$ and a pressure range up to $862 \mathrm{kPa}$. Subjects were instructed to position themselves directly on the mat with their feet a comfortable distance apart for each lift. Pressure data were recorded at $100 \mathrm{~Hz}$ and a $5 \mathrm{~V}$ square wave pulse facilitated synchronous collection of EMG, goniometry and planter pressure data.

\subsection{Statistical Analysis}

Descriptive statistics were performed on all data. Paired samples T-tests were used for data analysis to measure differences in paired muscle activation between the two lifts as well as Cohen's d to determine effect size. Statistical significance was set at $\mathrm{P} \leq 0.05$. SPSS 20.0 software (SPSS Inc., Chicago, IL) was used for all analyses.

\section{Results}

\subsection{Electromyography}

Group means (SD) average and peak EMG data for each lift are presented in figure 1 and table 1, respectively. Paired sample T-tests identified significant muscle activation differences between the overhead press and the push-press included ES and EO. Average and peak EMG for ES was significantly greater in push-press compared to overhead press. These differences were observed in the overall movement $(\mathrm{P}<0.001)$, and during both eccentric $(\mathrm{P}<0.001)$ and concentric phases of the movement $(\mathrm{P}<0.01)$. Additionally, during the eccentric phase, peak activity in EO was greater in push-press compared to overhead press $(\mathrm{P}<0.05)$.

Table 1. Average EMG data across the full range of movement, eccentric and concentric phases. Data are expressed as group mean (SD), normalized to percentage of MVIC $(\mathrm{mV})$.

\begin{tabular}{llll} 
& Push-press & Overhead Press & Effect Size (Cohen's $\boldsymbol{d}$ ) \\
\cline { 2 - 4 } Overall & $5.7(4.5)$ & $4.5(3.5)$ & 0.30 \\
RA & $13.9(9.1)$ & $12.5(16.2)$ & 0.11 \\
TA & $18.4(6.8)$ & $16.4(8.3)$ & 0.26 \\
EO & $32.2(11.5)^{* * *}$ & $11.4(9.1)$ & 2.0 \\
ES & & & \\
& & & \\
Eccentric & $3.7(3.4)$ & $5.3(3.7)$ & 0.45 \\
RA & $10.1(6.9)$ & $13.2(9.8)$ & 0.37 \\
TA & $14.7(6.3)$ & $19.2(9.8)$ & 0.55 \\
EO & $30.3(12.9)^{* * *}$ & $11.7(8.8)$ & 1.68 \\
ES & & & \\
& & & \\
Concentric & $6.5(6.3)$ & $3.7(3.6)$ & 0.54 \\
RA & $16.7(12.3)$ & $11.9(8.1)$ & 0.46 \\
TA & $21.1(9.5)^{*}$ & $13.9(7.6)$ & 0.83 \\
EO & $32.0(13.6)^{* * *}$ & $11.1(9.6)$ & 1.78 \\
ES & & & \\
\hline$*$
\end{tabular}

*significant differences between push and overhead press $(* \mathrm{P}<0.05 ; * * * \mathrm{P}<0.001)$. 


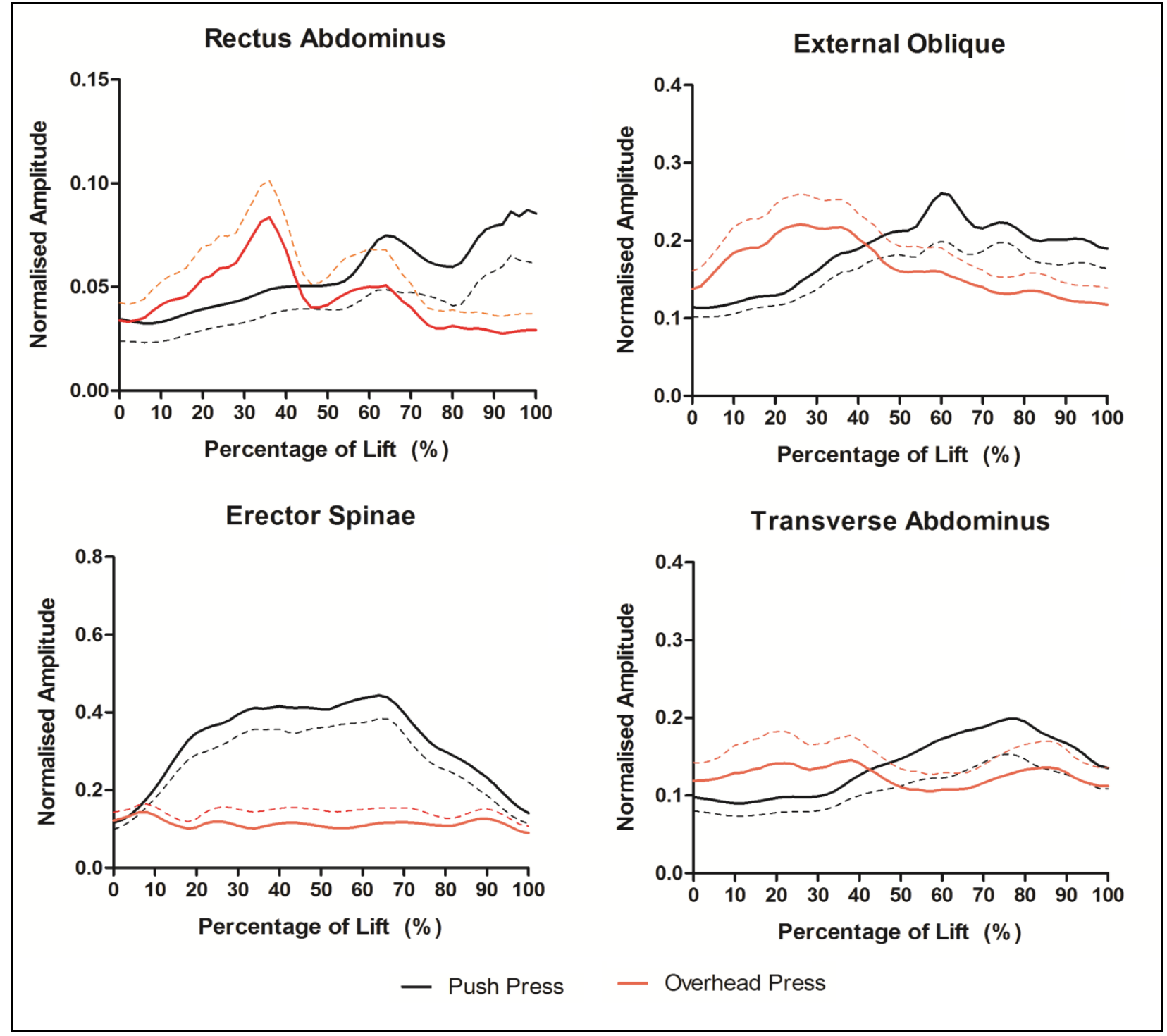

Figure 1. Group mean EMG ensembles for RF, EO, ES and TA during overhead press (red) and push-press (black) movements. Solid lines denote mean while dashed lines denote SEM.

\subsection{Center of Pressure}

Anterior displacement of COP was also not significantly different between push-press and overhead press for the overall movement $(21.8 \pm 6.4$ vs. $21.2 \pm 5.8 \mathrm{~cm})$, or during the concentric phase $(21.6 \pm 7.9$ vs. $24.7 \pm 7.7 \mathrm{~cm})$ (figure 2). However, during the eccentric phase, anterior displacement of COP was significantly greater in push-press compared to overhead press $(22.1 \pm 5.5$ vs. $17.5 \pm 4.8 \mathrm{~cm}, \mathrm{P}<0.05)$.

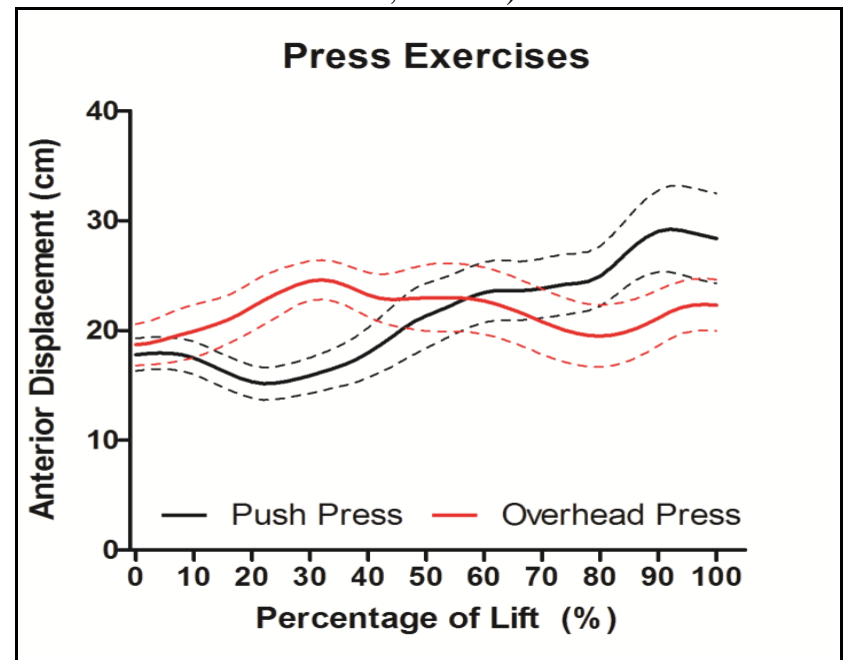

Figure 2. Group mean anterior displacement of COP data during press exercises. Solid lines denote mean while dashed lines denote SEM. 


\section{Discussions}

\subsection{Discussion overview}

Development of the core has become very popular in the training of both athletes and non-athletes. In the process of doing so, the use of unstable training has gained popularity based on research that identified increased core muscle activation during various free weight exercises (Anderson \& Behm, 2005, Norwood et al, 2007, Marshall \& Murphy, 2006). At the same time, commonly performed ground based exercises on a stable surface had been overlooked for their capacity to activate the muscles of the core. The purposes of this study was to determine the magnitude of core muscle activation generated during commonly performed ground-based lifts such as the overhead press and the push-press, and determine if one exercise variation generated greater core muscle activation than the other. An unstable surface is not necessary for activation of the core muscles when performing ground- based lifts (Hamlyn et al. 2007).

\subsection{EMG comparison}

Average EMG for all examined muscles was higher for the push-press compared to the overhead press. However, the greatest difference was seen with the ES for both peak EMG and average EMG during both the concentric and eccentric phases of the lift. The enhanced muscle activation of the ES is likely due to the greater dynamics of the push-press itself and the transfer of energy from the lower extremities to the upper extremities and the need to statically stabilize the spine as identified by Shinkle et al. (2012). The push-press also resulted in significantly greater anterior displacement of COP during the eccentric phase of the lift, despite identical resistance and the eccentric phases being relatively similar in movement between the two lifts. It is likely that adjustments in COP are required in the eccentric phase of the pushpress in order to compensate for differing lower limb kinematics in the initial concentric phase. Push-press EO EMG was also identified as superior when compared to the overhead press though only for peak EMG during the eccentric phase and not average EMG for the entire lift.

Willardson et al. (2009) also measured EMG activity on the RA, EO, TA, and ES during the overhead press while performed on a stable surface with 50\% and 75\% 1-RM and on a BOSU ball with 50\% 1-RM. Significantly greater RA and EO activation was observed in the stable condition with 75\% 1-RM when compared to the stable condition at 50\% 1-RM and 50\% 1-RM on the BOSU ball. TA activity in the stable condition with 75\% 1-RM was significantly greater than the 50\% BOSU ball condition only. No differences were identified with the ES between conditions. Kohler et al. (2010) compared EMG between RA, ES (lower and upper), and EO during the seated overhead press on a stable surface and unstable surface (Swiss ball) with both a barbell and dumbbells. Uribe et al. (2010) basically did the same thing but only observed the RA (of the core muscles) and only used dumbbells. Kohler et al. (2010) identified significant differences between the RA and lower ES during the unstable condition with a barbell when compared to the other conditions while Uribe found no differences in the RA between conditions. None of the above studies tested the pushpress thus further comparison with the current data is not possible.

\section{Conclusions and Practical Application}

Both of the ground-based exercises completed generated core muscle activation without the need of an unstable surface. However, the push-press was identified as superior in core muscle activation when compared to the overhead press. The average EMG of all 4 muscles was higher in push-press compared to overhead press, with the load being equal in both exercises. The push-press appears to be more effective at training the core musculature than the overhead press.

\section{Acknowledgements}

No funding was received for this study.

\section{References}

Akuthota, V, and Nadler, SF. (2004) Core strengthening. Archives of Physical Medicine and Rehabilitation, 85(3 suppl):S86-92.

Anderson, KG, and Behm, DG. (2004) Maintenance of EMG Activity and Loss of Force Output with Instability. Journal of Strength and Conditioning Research, 18, 637-640.

Anderson, K, and Behm, DG. (2005) Trunk muscle activity increases with unstable squat movements. Canadian Journal of Applied Physiology, 30:33-45.

Barnett, C. Kippers, V. Turner, P. (1995) Effects of Variations of the Bench Press Exercise on the EMG Activity of Five Shoulder Muscles. Journal of Strength and Conditioning Research, 9, 222-227.

Behm, DG, Drinkwater, EJ, Willardson, JM, and Cowley, PM. (2010) Canadian Society for Exercise Physiology position stand: The use of instability to train the core in athletic and nonathletic conditioning. Applied Physiology, Nutrition, and Metabolism, 35:109-112.

Bompa, TO. (1999) Periodization Training for Sports. Champaign, IL: Human Kinetics.

Byrne, JM, Bishop, NS, Caines, AM, Crane, KA, Feaver, AM, and Pearcey, GEP. (2014) Effect of using s suspension training system on muscle activation during the performance of a front plack exercise. Journal of Strength and Conditioning Research, 28:3049-3055. 
Gullett, JC, Tillman, MD, Gutierrez, GM, and Chow, JW. (2009) A biomechanical comparison of back and front squats in healthy trained individuals. Journal of Strength and Conditioning Research, 23, 284-292.

Gamble, P. An integrated approach to training core stability. (2007) Strength and Conditioning Journal, 29 , 58-68.

Hermens, H, Freriks, B, Disselhorst-Klug, C, Rau, G. (2000) Development of recommendations for SEMG sensors and sensor placement procedures. Journal of Electromyography and Kinesiology, 10: 361-374.

Kibler, BW, Press, J, Sciascia. (2006). The role of core stability in athletic function. Sports Medicine, 36(3):189-198.

Kohler, JM, Flanagan, SP, and Whiting, WC. (2010) Muscle activation patterns while lifting stable and unstable loads on stable and unstable surfaces. Journal of Strength and Conditioning Research, 24, 313-321.

Leetun, DT, Ireland, ML, Willson, JD, Ballantyne, BT, and Davis, IM. (2004) Core stability measures as risk factors for lower extremity injury in athletes. Medicine and Science in Sport and Exercise, 36, 926-934.

Marshall, PWM, and Murphy, BA. (2006) Increased deltoid and abdominal muscle activity during swiss ball bench press. Journal of Strength and Conditioning Research, 20: 745-750.

Mayhew, JL, Bird, M, Cole, ML, Koch, AJ, Jacques, JA, Ware, JS, Buford, BN, and Fletcher, KM. (2005) Comparison of the backward overhead medicine ball throw to power production in college football players. Journal of Strength and Conditioning Research, 19: 514-518.

McGill, SM. (2009) Ultimate Back Fitness and Performance. (4 ${ }^{\text {th }}$ ed.). Waterloo, Ontario, Canada: Wabuno Publishers.

McGill, SM, Grenier, S, Kavcic, N, Cholewicki, J. (2003) Coordination of muscle activity to assure stability of the lumbar spine. Journal of Electromyography and Kinesiology, 13:353-359.

Norwood, JI, Anderson, GS, Gaetz, MB, and Twist, PW. (2007) Electromyographic activity of the trunk stabilizers during stable and unstable bench press. Journal of Strength and Conditioning Research, 21:343-347.

Parkhouse, KL. and Ball, N. (2011) Influence of dynamic versus static core exercises on performance in field based fitness tests. Journal of Bodywork and Movement Therapies, 15:517-524.

Saeterbakken, AH, and Fimland, MS. (2013) Muscle force output and electromyographic activity in squats with various unstable surfaces. Journal of Strength and Conditioning Research, 27:130-136.

Shinkle, J, Nesser, TW, Demchak, TM, and McMannus, DM. (2012) Effect of Core Strength on the Measure of Power in the Extremities. Journal of Strength and Conditioning Research,25:373-380.

Snarr, RL and Esco, MR. (2014) Electromyographical comparison of plank variations performed with and without instability devices. Journal of Strength and Conditioning Research, 28:3298-3305.

Uribe, BP, Coburn, JW, Brown, LE, Judelson, DA, Khamoui, AV, and Nguyen, D. (2010) Muscle activation when performing the chest press and shoulder press on a stable bench vs. a Swiss ball. Journal of Strength and Conditioning Research, 24, 1028-1033.

Willardson, J. Core stability training: applications to sports conditioning programs. (2007) Journal of Strength and Conditioning Research, 21:979-985.

Willardson, J, Fontana, FE, Bressel, E. (2009) Effect of surface stability on core muscle activity for dynamic resistance exercises. International Journal of Sports Physiology Performance, 4:97-109. 